University of Wollongong

Research Online

Faculty of Engineering and Information

Faculty of Engineering and Information

Sciences - Papers: Part B

Sciences

2018

Position control of a soft prosthetic finger with limited feedback information

Solen Kumbay-Yildiz

Hacettepe University, solen@uow.edu.au

Rahim Mutlu

University of Wollongong, rmutlu@uow.edu.au

Gursel Alici

University of Wollongong, gursel@uow.edu.au

Follow this and additional works at: https://ro.uow.edu.au/eispapers1

Part of the Engineering Commons, and the Science and Technology Studies Commons

Research Online is the open access institutional repository for the University of Wollongong. For further information contact the UOW Library: research-pubs@uow.edu.au 


\title{
Position control of a soft prosthetic finger with limited feedback information
}

\author{
Abstract \\ One of the challenging research questions in soft robotics is the establishment of valid mathematical \\ models and control techniques under limited feedback conditions. A soft robotic system will typically \\ have a continuum or monolithic body seamlessly embodying its actuation and sensing elements, \\ accompanied by mechanical structure, and even the energy source, much like a biological system. In this \\ study, we propose a relay sliding mode controller based on the input - output model (RSMC-IO), which is a \\ simple but effective control approach that does not require a complete system model, to control the tip \\ position of a soft prosthetic finger, which typifies a monolithic soft robotic positioning system. The \\ feedback signal corresponding to the bending angle of each joint of the finger is provided by highly \\ stretchable soft strain sensors made of a silicone (EcoFlex 0010) substrate containing channels filled \\ with carbon paste as the conductive component. An analytical expression between the fingertip and joint \\ angles of the finger is derived and experimentally verified. This expression is accurate enough to estimate \\ the joint positions (i.e. joint angles) as well as the fingertip. The experimental results show the efficacy of \\ the proposed controller, which has not been proposed for prosthetic fingers and similar soft robotic \\ positioning systems before.

\section{Disciplines} \\ Engineering | Science and Technology Studies

\section{Publication Details} \\ Kumbay-Yildiz, S., Mutlu, R. \& Alici, G. (2018). Position control of a soft prosthetic finger with limited \\ feedback information. Proceedings of the 2018 IEEE/ASME International Conference on Advanced \\ Intelligent Mechatronics (AIM) (pp. 700-705). United States: IEEE.
}




\title{
Position Control of a Soft Prosthetic Finger with Limited Feedback Information*
}

\author{
S. Kumbay Yildiz, Member, IEEE, R. Mutlu, and G. Alici
}

\begin{abstract}
One of the challenging research questions in soft robotics is the establishment of valid mathematical models and control techniques under limited feedback conditions. A soft robotic system will typically have a continuum or monolithic body seamlessly embodying its actuation and sensing elements, accompanied by mechanical structure, and even the energy source, much like a biological system. In this study, we propose a relay sliding mode controller based on the input - output model (RSMC-IO), which is a simple but effective control approach that does not require a complete system model, to control the tip position of a soft prosthetic finger, which typifies a monolithic soft robotic positioning system. The feedback signal corresponding to the bending angle of each joint of the finger is provided by highly stretchable soft strain sensors made of a silicone (EcoFlex 0010) substrate containing channels filled with carbon paste as the conductive component. An analytical expression between the fingertip and joint angles of the finger is derived and experimentally verified. This expression is accurate enough to estimate the joint positions (i.e. joint angles) as well as the fingertip. The experimental results show the efficacy of the proposed controller, which has not been proposed for prosthetic fingers and similar soft robotic positioning systems before.
\end{abstract}

\section{INTRODUCTION}

Robotic prosthetics, despite not being a new field of research, continues to be an important field on which a significant amount of research has been undertaken recently. Particularly, upper limb robotic prosthetics has gained great potential and interest among researchers in last a few years. Functionality, comfort and cost may be regarded as the three common and most important aspects of prosthetics. The field of soft robotics appears to hold the key toward realising prosthetic structures with various functions, made entirely from soft and smart materials. These soft robotic prostheses, unlike their conventional counterparts, are not to be made of rigid parts, will consequently be lightweight, require little or no assembly and therefore, come at a lower production cost.

*Research supported by the Intelligent Nano-Tera Research Systems Laboratory and by ARC Centre of Excellence for Electromaterials Science (Grant No. CE140100012).

S. Kumbay Yildiz is with the Department of Electrical and Electronics Engineering, Hacettepe University, 06800, Ankara, Turkey (e-mail: solen@ee.hacettepe.edu.tr).

R. Mutlu is with the School of Mechanical, Material, Mechatronic and Biomedical Engineering, University of Wollongong, Wollongong, NSW, 2522, Australia and the ARC Centre of Excellence for Electromaterials Science, University of Wollongong, Wollongong, NSW, 2522, Australia (e-mail: rmutlu@uow.edu.au).

G. Alici is with the School of Mechanical, Material, Mechatronic and Biomedical Engineering, University of Wollongong, Wollongong, NSW, 2522, Australia and the ARC Centre of Excellence for Electromaterials Science, University of Wollongong, Wollongong, NSW, 2522, Australia (corresponding author, phone: +61 24221 4145; fax: +61 24221 3101; e-mail: gursel@uow.edu.au).
The challenges of developing a soft prosthetic structure are twofold. The first challenge involves replacing the two key functions of such a structure, namely sensing and actuation, with their soft counterparts. In the past decade, a great deal of progress has been made in developing soft sensors and actuators from electroactive polymers (EAP) to artificial muscles (PAM) [1-3]. Change of certain electrical properties due to the change in device geometry in response to an applied mechanical stimulus constitutes the basis for soft sensors [4-7]. Modelling and control constitute the other major challenge in soft robotics [8]. Unlike conventional rigid robots, soft robots do not have widely agreed models. The difficulty arises from the fact that soft robotic structures, just like the natural systems they aim to mimic or represent, can accomplish highly complex and continuous motions, such as bending, stretching or twisting. The lack of a well-defined number of degrees of freedom a soft robotic body has due to these complex movements, and that of a well-understood model makes the control of such structures extremely arduous [9-11].

As part of the ongoing efforts in soft robotics, we recently conducted a comparative study on microfluidic silicone strain sensors and reported on their fabrication and response characterisation [12]. We also discussed their potential employment on a soft robotic prosthetic finger. We concluded that the combination of EcoFlex and liquid carbon black would be preferred due to fast transient characteristics and slightly longer durability. We put the sensors made from this particular combination of materials to test by comparing the sensor's performance with that of a conceptually different, commercially available soft strain sensor [13]. While both sensors have similar linearity and measurement resolution properties, the commercial sensor displays large hysteresis due to its slow transient response, which makes the microfluidic strain sensor preferable in soft robotics applications, especially when the input stimuli change in a step-wise manner. The commercial sensor has the advantage of remaining operational for a longer period of time. In this paper, we aim to take advantage of the results in our previous studies and achieve an accurate position control of the tip of a soft prosthetic finger by relying on the feedback received from our soft sensors. A number of recent studies can be regarded as partly relevant to our work, as they focus on developing gloves, mainly for rehabilitation purposes, that are used for hand gesture and posture monitoring [14-16]. Other works such as $[17,18]$ concentrate their efforts on designing and manufacturing soft robotic hands made from compliant materials. These studies, however, do not elaborate on the control of these structures. Some works rely on a minimal predefined control action based on preliminary observations [19]; others aim to obtain a more detailed mathematical model 
of the robotic element's behaviour and use a control scheme based on feedback from external sensors such as cameras [20].

In this study, a soft strain sensor is placed on each joint of an underactuated 3D printed soft prosthetic finger, where the highest strain rates are observed using the finite element method [21]. Its joint and tip positions are extrapolated from the sensor data as the finger is flexed and extended. The position of the fingertip is controlled using only the feedback from these sensors; no external sensor data is used, much like a continuum body containing its sensors in its mechanical structure. Since obtaining a detailed model of such a system is not practical, we have proposed a relay sliding mode controller based on the input - output model (RSMC-IO), which is a simple but effective control approach that does not require a complete system model, thus presenting a significant advantage [22]. The contributions of this study are (1) the incorporation of highly stretchable and durable soft strain sensors made of carbon paste with linear response into a soft prosthetic finger and (2) application of a simple and effective closed-loop control strategy to a soft robotic structure with limited feedback information.

The organisation of the paper is as follows. The introduction is followed by a description of materials used in this study. Section 3 summarises the experimental setup and preliminary steps that are taken before closed-loop control is applied. The control algorithm is also explained in this section. The results of our experiments and the discussion of data are presented in Section 4. Section 5 contains our concluding remarks and elaborates on future studies.

\section{DESCRIPTION OF MATERIALS}

\section{A. 3D printed soft prosthetic finger}

The soft prosthetic finger used in our experiments has a fully compliant monolithic structure fabricated by employing a fused deposition modelling (FDM) based additive manufacturing method with the help of a $3 \mathrm{D}$ printer and a thermoplastic polyurethane (TPU) material [21,24,25]. The 3D printer (UP Plus 2, 3D Printing Systems) uses a modified extruder unit and customised printing parameters in order to successfully extrude the TPU material. The design of the finger includes flexure hinges instead of revolute joints which allows the structure to be printed as a whole (i.e. monolithic) from a single material and a single printing process. The finger is an underactuated system with three degrees of freedom and only one input, and with anthropomorphic dimensions of a human index finger [26]. Its actuation is accomplished by a tendon cable pulled or released by a DC motor. Figure 1 shows the finger used in our experiments and indicates the names of the individual joints (metacarpal phalangeal joint - MCP, proximal interphalangeal joint - PIP and distal interphalangeal joint - DIP) and the fingertip, which is the main point of interest.

\section{B. Highly stretchable soft sensors}

We used highly stretchable elastomeric strain sensors that are fabricated using Smooth-On's EcoFlex 0010 as base substrate and carbon paste (Carbon Conductive Grease, MG Chemicals) as the conductive component. EcoFlex 0010 is an extremely soft, low-viscosity two component, addition cured,

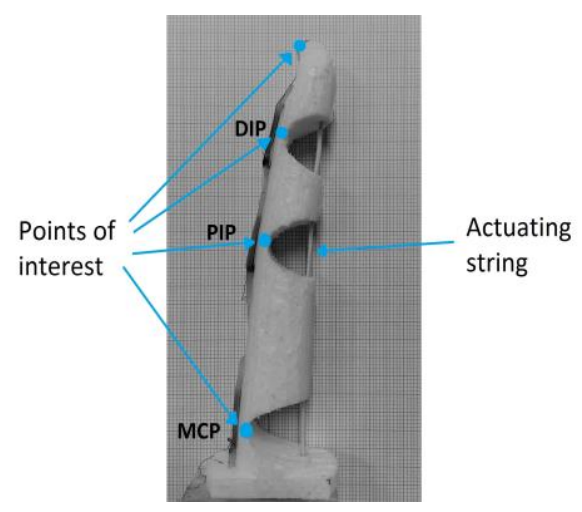

Figure 1. 3D printed soft prosthetic finger.

room temperature vulcanising (2 RTV) silicone rubber. The fabrication steps of these sensors highly resemble those of the EcoFlex sensors in [12] with the exception of channel geometry and conductive material filled into the channels; therefore, the details of their fabrication are not presented here. Three identical sensors are fabricated for the experiments. A finished sensor can be seen in Figure 2. The total thickness of a sensor is $1 \mathrm{~mm}$, the U-shaped pattern is 15 $\mathrm{mm}$ long, and the channels are $0.6 \mathrm{~mm}$ high and $1.2 \mathrm{~mm}$ wide. We have recently spray coated similarly shaped soft strain sensors with a thickness of $500 \mathrm{~nm}$ made of styrenebutadiene-styrene and multi-walled carbon nanotube ink on the same monolithic finger [27].

In our previous work, we fabricated soft strain sensors having a strain gauge type geometry and with various combinations of different base substrates, one of which was EcoFlex 0010, and conductive fluids [12]. Through several tests we observed that the sensors are capable of providing reliable and almost linear strain measurement data. We also concluded that EcoFlex is favourable for applications in which the input stimulus changes at a fast rate due to its fast response characteristics when it is both stretched and released. In addition, the yield strength of the EcoFlex is higher in comparison to that of the TPU material used for 3D printing the finger. It means that the soft strain sensor does not show plastic deformation, which directly contributes to the linearity of the strain data. However, all sensors fabricated during this study suffered from the same problem; the conductive liquids were absorbed into the base substrate (silicone matrix), thus rendering the sensors inoperable in a few hours after fabrication. To overcome this problem, we simplified the pattern geometry and increased its dimensions, so that the channels can be filled with carbon paste; a more viscous material than the conductive fluids used in the previous studies. According to our observations, the sensors stay operational for at least several months after fabrication, since carbon paste does not appear to be absorbed into the silicone matrix.

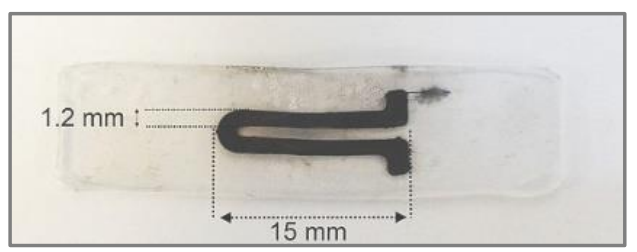

Figure 2. A finished soft strain sensor and pattern dimensions. 


\section{EXPERIMENTAL SETUP, MODEL AND CONTROL RESUlTS}

\section{A. Experimental apparatus}

The soft stretchable strain sensors described in the previous section are carefully placed onto the flexure hinges of the prosthetic finger as shown in Figure 3. The prosthetic finger is then mounted sideways on a stationary platform with its base anchored on the platform. The end of the actuation string is fixed on a translation stage which is driven by a DC motor. As the micrometre equipped translation stage is moved using the motor, the string is either pulled or released, thereby flexing or extending the finger depending on the direction of motion. The electrical responses of the sensors are recorded by means of a basic voltage divider circuit. A DC voltage source and a known resistor are connected in series to each sensor and the voltage drop on the known resistors are recorded on a personal computer equipped with a data acquisition (DAQ) card (NI 6251, National Instruments). This setup can be seen in Figure 4. The control algorithm used in the applications is developed using MATLAB (The MathWorks, Inc.) and embedded inside a Simulink model. This model uses the Real-Time Windows Target Blockset along with the DAQ card to receive the electrical responses from the sensors and calculate the current position of the fingertip. It also determines the correct control action and applies the corresponding output voltage to the DC motor. A high resolution camera is placed perpendicular to the motion plane of the finger directly above the stationary plate on which the finger is mounted and used to record the movement of the finger during the applications. With the image processing tools of MATLAB, images taken by the camera are analysed frame by frame to extract the position of the points of interest, i.e. joints and the fingertip during the preparatory stages of the experiments as explained in the following subsection. Once again, it is important to emphasise that information received from the camera is not in any way used in the control strategy; it is only used to verify the efficacy of the control algorithms.

\section{B. Relation between sensor data and fingertip position}

Before attempting closed-loop control, a preliminary data collection cycle is needed to determine the relationship between the information gathered from the sensors and the corresponding fingertip position. To that end, the translation stage is moved to 13 predetermined locations and the sensors' electrical responses are recorded at these positions, while the positions of the joints and the fingertip are captured with the camera at the same time. Each position corresponds to a $3 \mathrm{~mm}$ displacement of the actuating string along the y-axis; the first 7 positions lie on the forward path as shown in Figure 5a, i.e. when the finger is flexed, and the remaining 6 are on the backward path, i.e. when the finger is relaxed. The exact displacement of the string is achieved with the help of the micrometre fixed to the translation stage.

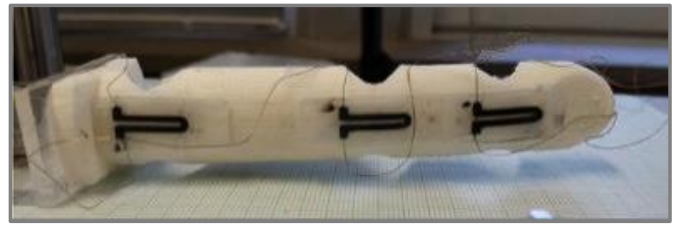

Figure 3. Side view of the prosthetic finger operating in horizontal plane with sensors fixed on flexure hinges.

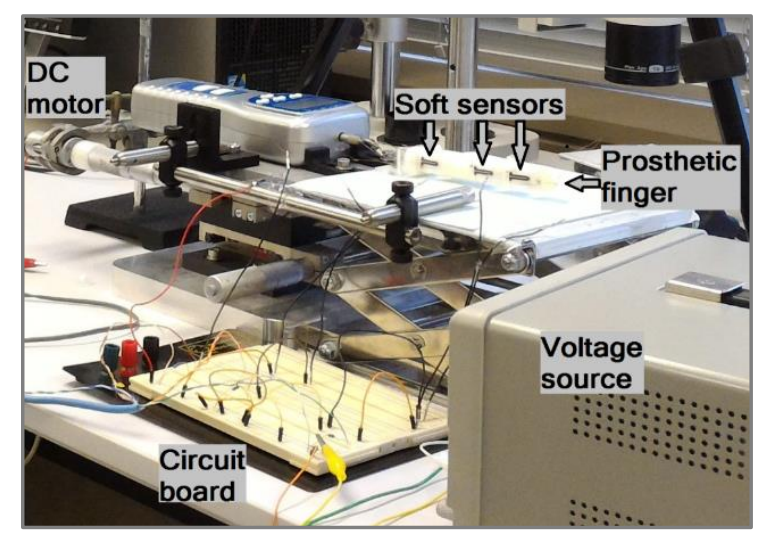

Figure 4. Measurement setup.

The resistance value of each sensor $R_{p i}$ (resistance of the sensor placed on the $i^{\text {th }}$ joint at position $p$ ) and the change of resistance $\left(\Delta R_{p i} / R_{0 i}\right)$, are calculated for each position. Using the image processing tools of MATLAB, the individual positions of each joint and the fingertip on the $\mathrm{x}-\mathrm{y}$ plane are extracted from the images taken during the experiment described above. Then the joint angles $\left(\theta_{p i}\right)$, as shown in Figure $5 \mathrm{~b}$, are calculated. Note that the joint angles are not equal to zero when the finger is at rest $\left(\theta_{0 i} \neq 0\right)$. Therefore, instead of finding a relation between the actual joint angles and change of resistance values, a relation for the deviation of the joint angles $\left(\Delta \theta_{p i}\right)$ from their initial values is estimated ( $p=0,2, \ldots, 12$ and $i=1,2,3$.). The change of resistance values and angle deviations are shown in matrix form in Eq. 1 as

$$
\Delta \boldsymbol{\theta}=\left[\begin{array}{ccc}
0 & 0 & 0 \\
\Delta \theta_{11} & \Delta \theta_{12} & \Delta \theta_{13} \\
\vdots & \vdots & \vdots \\
\Delta \theta_{p 1} & \Delta \theta_{p 2} & \Delta \theta_{p 3}
\end{array}\right], \Delta \mathbf{R}=\left[\begin{array}{ccc}
0 & 0 & 0 \\
\frac{\Delta R_{11}}{R_{01}} & \frac{\Delta R_{12}}{R_{02}} & \frac{\Delta R_{13}}{R_{03}} \\
\vdots & \vdots & \vdots \\
\frac{\Delta R_{p 1}}{R_{01}} & \frac{\Delta R_{p 2}}{R_{02}} & \frac{\Delta R_{p 3}}{R_{03}}
\end{array}\right]
$$

where $\Delta \theta_{p i}=\theta_{p i}-\theta_{0 i}$ and $\Delta R_{p i}=R_{p i}-R_{0 i}$. Here, $\theta_{0 i}$ and $R_{0 i}$ denote the initial values of the $i$ th joint angle and the initial value of the sensor placed on the same joint. Note that the angle values in each row of $\Delta \boldsymbol{\theta}$ in conjunction with the link lengths can be used to determine the fingertip position of the finger on the $x-y$ plane at any instance with the help of basic trigonometry. Assuming a linear relationship between
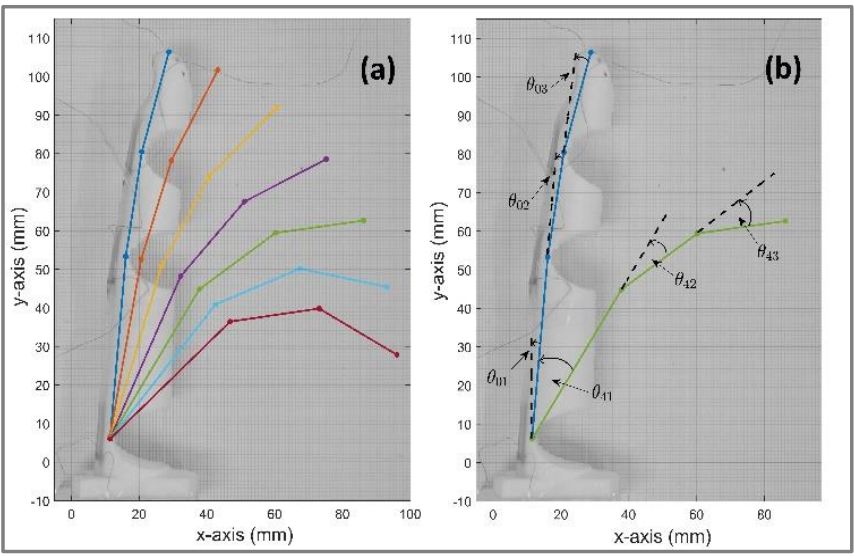

Figure 5. (a) The first 7 predetermined positions and (b) joint angles at the initial ( $\mathrm{p}=0$, at rest) and the 5th position $(\mathrm{p}=4)$. 
the change of resistance and the angle deviation, we can indicate this relation with

$$
\Delta \boldsymbol{\theta}=\Delta \mathbf{R K}
$$

where $\mathbf{K}$ is a $3 \times 3$ matrix which can be found using the least squares approach as

$$
\mathbf{K}=\left(\Delta \mathbf{R}^{\mathrm{T}} \Delta \mathbf{R}\right)^{-1} \Delta \mathbf{R}^{\mathrm{T}} \Delta \boldsymbol{\theta}
$$

The goodness of the estimation of $\mathbf{K}$ is depicted in Figure 6 . Figure 6a shows the fingertip positions calculated using the values in $\Delta \boldsymbol{\theta}$, whereas Figure $6 \mathrm{~b}$ contains both the actual positions shown in Figure 6a and the estimated fingertip positions which are simply calculated using the estimated angle values that are simply found from

$$
\Delta \boldsymbol{\theta}_{\text {est }}=\Delta \mathbf{R K} \text {. }
$$

According to Figure $6 a$, the trajectories traced by the joints and the tip of the prosthetic finger are slightly different during the flexing and extending phases of the finger's motion. This, so called, hysteresis in its behaviour is a commonly encountered phenomenon in soft structures. Figure $6 \mathrm{~b}$ indicates that the matrix $\mathbf{K}$ provides a sufficiently accurate estimation of joint angle deviations and thereby also positions for the joints, as well as the fingertip.

\section{Closed-loop control of the prosthetic finger}

The objective of the experiments is to control the fingertip position of the prosthetic finger on the $\mathrm{x}-\mathrm{y}$ plane. The system is considered as a single input single output system, where the output variable is the distance of the fingertip from the MCP joint. Note from Figure 6 that the displacement of this joint is negligibly small; therefore, its position is considered constant during the motion of the finger. The input variable is the voltage applied to the DC motor which pulls and releases the actuating string. At each sampling instant, the readings from the sensors are used to calculate the joint angles, which are then used to calculate the distance of the fingertip from the MCP joint, i.e. the output. Depending on the set-point chosen by the designer and the current position of the fingertip, the control algorithm calculates the necessary control action to the DC motor.

The control scheme we chose for this study is the relay sliding mode control based on the input-output model (RSMCIO) $[22,23]$. RSMC-IO is an easy to apply, yet very effective robust control method proposed for relay controlled systems. The objective is to force the relay element to operate in sliding

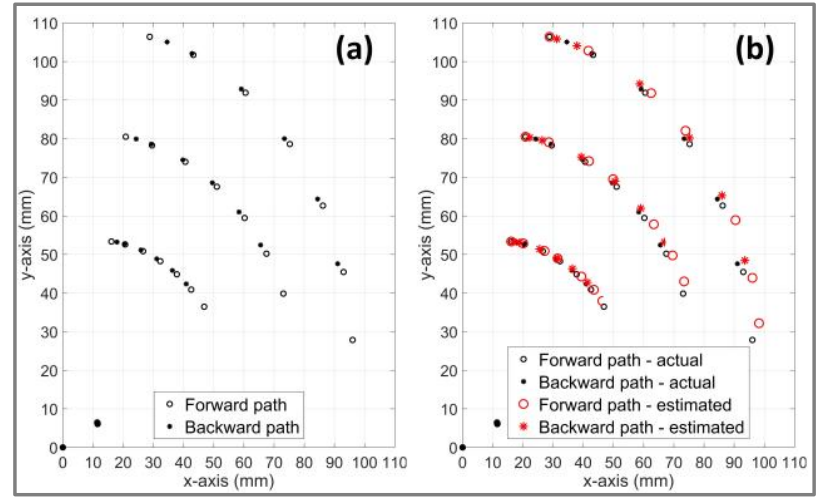

Figure 6. Goodness of the position estimation: (a) Actual joint and fingertip positions, (b) actual and estimated values using $\mathrm{K}$. mode in order to obtain a robust control performance against parameter variations and disturbances. While SMC methods are traditionally defined in state-space, RSMC-IO is formulated using the input-output description of the system; therefore, it can be applied without the need to know or measure the system states. Moreover, a complete system model is not necessary; the only a priori knowledge required is the relative degree of the open-loop transfer function. Figure 7 shows the block diagram of a single variable relay system where $G(s)$ is the transfer function of the open-loop system and $M(s)$ is the transfer function of the desired model. $w, e, u$ and $y$ represent the set-point (or reference signal), relay input (also referred to as the error), control input and closedloop system output, respectively. $\emptyset$ denotes the system's output filtered with the inverse of the model. $s$ is either the differential operator $(d / d t)$ or the Laplace operator depending on the context. The relay element is assumed to be symmetric and ideal with the amplitude $r$. The control law is given by

$$
u=r \operatorname{sign}(e)=\left\{\begin{array}{rr}
r, & e \geq 0 \\
-r, & e<0
\end{array} .\right.
$$

The necessary and sufficient condition for sliding motion to occur in this system is given by the inequality

$$
e \dot{e}<0
$$

which basically means that, when the relay input $e$ crosses the threshold $(e=0)$, it immediately recrosses it, resulting in sliding motion. The system is then said to operate in sliding mode and the closed-loop system output will only be governed by the reference model.

$$
e=w-M^{-1} y=0 \quad \Rightarrow y=M w
$$

It is important to point out that the loop transfer function of the system shown in Figure 7 must satisfy the condition

$$
\operatorname{rel}\left(M^{-1} G\right)=1
$$

for sliding motion to occur, where rel indicates relative degree. Eq. 10 indicates that the relative degree of the system to be controlled needs to be known to adjust the relative degree of the loop transfer function by choosing a reference model of appropriate order. Note that, relative degree is the difference between the degree of the denominator polynomial and the degree of the numerator polynomial of the transfer function. One way of determining the relative degree of a system, whether it is linear or nonlinear, is to obtain its open-loop response to a non-zero step input first and then find the number of times the output expression has to be differentiated until the input appears in the expression of the differentiated output. In order to determine the relative order of the system indicated as $G(s)$ in Figure 7, we examined the individual responses of the sensors placed on finger joints. Figure 8 shows the resistance changes of each sensor as a result of a square wave shaped input. The output of a system with a relative order of 1 has a nonzero slope at the time of the step input. Clearly, this is the

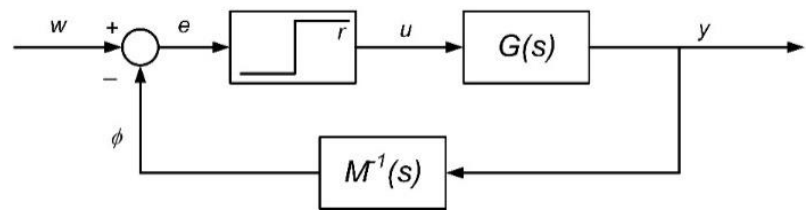

Figure 7. A relay controlled system. 
case with all three sensor responses seen in Figure 8. To further prove this claim, each graph in Figure 8 also contains the response of a linear parametric model of relative order 1 , estimated using a recursive least squares approach, indicated with red dashed lines. The parameters of these models are not provided here, since the models themselves are not needed in the control algorithm, only the relative order is of interest. In brief, we can conclude that each sensor response can be represented by a transfer function with the relative order 1 . Since the distance of the fingertip is a linear combination of all three sensor responses, the overall open-loop system $G(s)$ can also be represented by a transfer function with the relative order 1. Since $\operatorname{rel}(G)=1$, Eq. 10 is satisfied without the inclusion of a reference model in the algorithm (i.e. $M(s)=$ $1)$, meaning that the output is required to follow the reference position without any other specifications regarding its transient characteristics. The relay amplitude $r$ is chosen as 10 , which is the maximum voltage that can be supplied by the DAQ card without applying external amplification. The discontinuous nature of the control leads to a phenomenon known as chattering which causes undesired effects such as low control accuracy and wearing of system elements and parts. In theory, the relay is assumed to switch at an infinite rate, causing no chattering. In practice, the frequency of switching is determined by the choice of the sampling interval. The sampling interval needs to be determined by taking into account the physical constraints on the actuator, in this case the DC motor. A sampling interval of $0.5 s$ is chosen, as the DC motor cannot respond any faster, so it is a restriction caused by the actuator which is used in an on-off fashion to mimic a relay element.

\section{EXPERIMENTAL RESULTS AND DISCUSSION}

Figure 9 presents the results of the closed-loop position control experiments with the prosthetic finger. Figure 9a shows the reference (dashed line) and actual distance (solid line) of the fingertip which is calculated based on the feedback

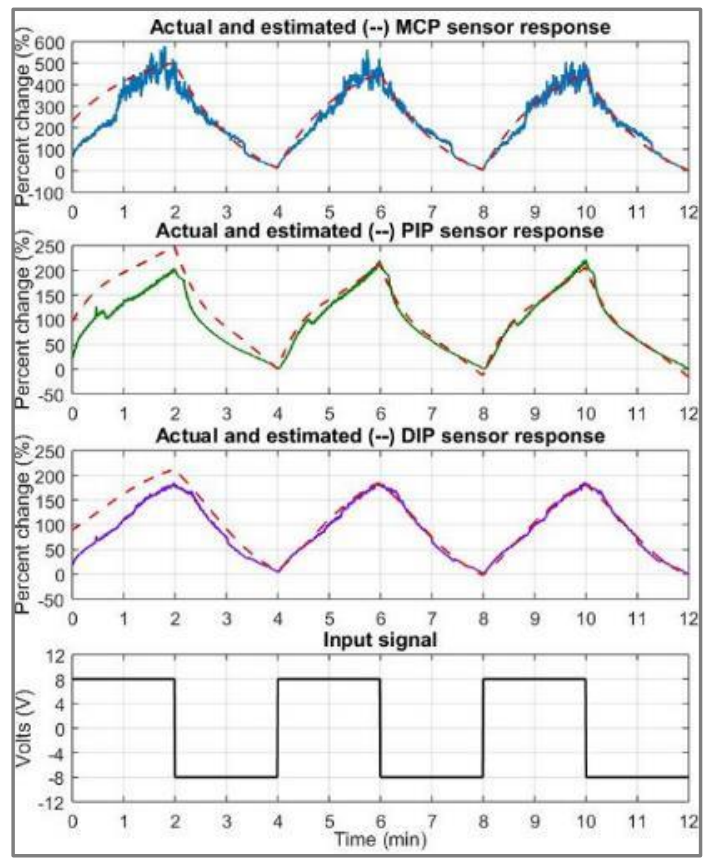

Figure 8. Responses of soft sensors and fitted models. from the soft strain sensors that are placed on the finger joints. The control input and the error are shown in Figure 9b and Figure 9c, respectively. The change of resistances of individual sensors are plotted in Figure 9d. Figure 9a shows that the output appears to reach and then follow the set-point well without an overshoot. Here, it is worth reemphasising that the output signal is calculated from the sensor data using Eq. 6 and the forward kinematic model of the finger shown in Figure 5a. Our observations indicate that the variation between this signal and the actual fingertip position observed through the camera above is within the range of $( \pm 2 \mathrm{~mm})$, resulting in an acceptable control accuracy. Figure $9 \mathrm{~b}$ shows that the system enters and continues to operate in sliding mode, i.e. the control input oscillates between the minimum and maximum relay amplitudes at a high frequency. It is said that the relay element, in this case the DC motor, is operating in sliding mode. This can also be observed by investigating the error in Figure 9c. Note that during sliding motion, the error $e$ is a signal with high frequency and low amplitude oscillating around zero. The only times sliding motion ceases is when the set-point changes; however, as soon as the output reaches the set-point, sliding motion is achieved again. Recall that the only feedback in this control system is provided by the soft sensors on the joints of the prosthetic finger. According to Figure 9d, the sensor placed on the MCP joint undergoes the most severe deformation; when the finger reaches the lowest set-point, there is almost a $200 \%$ increase in the sensor's resistance, whereas for the other two sensors this value remains below $100 \%$. The higher deformation level of the MCP sensor is also thought to contribute to higher noise levels observed in its output.

\section{CONCLUSION AND FUTURE STUDIES}

We have presented an effective methodology for the closed-loop control of the tip position of a soft prosthetic finger, representing a soft robotic system. The control scheme runs on the feedback data received from soft strain sensors

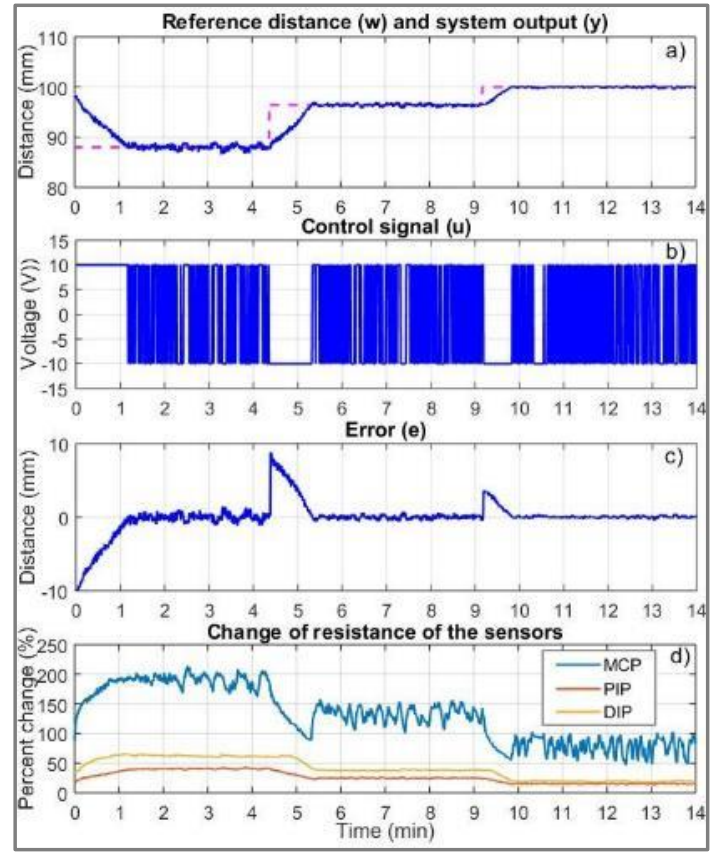

Figure 9. Closed loop control of the soft robotic prosthetic finger. 
placed on the joints of the prosthetic finger; no other physical feedback is utilised. Unlike traditional SMC strategies, the proposed controller is formulated using the input-output description of the system; eliminating the need to know or measure system states. Moreover, a complete system model is not necessary; only the relative degree of the open-loop system is needed. This is a significant advantage for soft robotic systems, for which it is not straightforward to establish a valid mathematical model.

The soft strain sensors are composed of channels trapped between two thin layers of a commercially available silicone compound. Resistance measurements provided by the sensors enable us to calculate each joint's angle and thus the position of the fingertip. While the fabrication technique used in this study is very similar to that in our previous studies, the channel geometry of the sensors is different. Moreover, a different material, carbon paste, is used as the filling material to provide the electrical properties of the sensors. The sensors in this study did not show any signs of the liquid absorption problem we reported on previously. In order to fill the channels with carbon paste, the channel dimensions were increased and the complexity of the channel geometry was reduced. Note that, while the change in the channel geometry is somewhat compromising the sensor's measurement resolution, it dramatically improves the repeatability of its operation and thus the sensor's usefulness.

The experimental setup enables us both to collect open loop data to establish sensor characteristics and run a closed loop control algorithm and display current progress on a computer screen. As expected from our previous studies, our experiments have shown that the readings from the sensors placed on the joints vary linearly with respect to joint angles. This linear relation is utilised to interpret the current position of the tip of the prosthetic finger. The results regarding the control phase indicate that our approach provides a feasible control for the fingertip position. The fingertip moves to desired positions within reasonable margins each time a new set-point is provided by the user. Using RSMC-IO, we were able to obtain a successful control performance without a detailed mathematical model representing the system.

Our future efforts will focus on improving the overall control performance of the soft robotic prosthetic finger by refining the current control algorithm and exploring various other control strategies, and comparing their performances with current. Ultimately, we will apply our findings to the position control of individual fingers of a complete soft robotic prosthetic hand. Towards that aim, we will continue to work on the design of the soft strain sensors, as well as testing different material combinations and the sensors' placement on the finger joints. Improving the overall sensing capability will fundamentally result in a better control accuracy.

\section{REFERENCES}

[1] C. Ching-Ping and B. Hannaford, "Measurement and modeling of McKibben pneumatic artificial muscles," IEEE Transactions on Robotics and Automation, vol. 12, pp. 90-102, 1996.

[2] B. Tondu, "Modelling of the McKibben artificial muscle: A review," Journal of Intelligent Material Systems and Structures, vol. 23, pp. 225253, 2012.

[3] R. Mutlu, G. Alici, and W. Li, "Electroactive polymers as soft robotic actuators: Electromechanical modeling and identification," in 2013
IEEE/ASME International Conference on Advanced Intelligent Mechatronics, 2013, pp. 1096-1101.

[4] Y. N. Cheung et al., "A novel fluidic strain sensor for large strain measurement," Sensors and Actuators A: Physical, vol. 147, pp. 401408, 2008.

[5] Y. L. Park et al., "Hyperelastic pressure sensing with a liquid-embedded elastomer," Journal of Micromechanics and Microengineering, vol. 20, p. 125029, 2010.

[6] Y.-L. Park, B.-R. Chen, and R. J. Wood, "Design and Fabrication of Soft Artificial Skin Using Embedded Microchannels and Liquid Conductors," Sensors Journal, IEEE, vol. 12, pp. 2711-2718, 2012.

[7] J. T. Muth et al., "Embedded 3D Printing of Strain Sensors within Highly Stretchable Elastomers," Advanced Materials, vol. 26, pp. 63076312, 2014.

[8] B. Trimmer, "Soft robot control systems: A new grand challenge?" Soft Robotics, vol. 1, pp. 231-232, 2014.

[9] M. Luo et al. "Towards modular soft robotics: Proprioceptive curvature sensing and sliding-mode control of soft bidirectional bending modules," Soft Robotics, vol. 4, pp. 117-125, 2017.

[10] W. Felt, K. Y. Chin, and C. D Remy, "Smart Braid Feedback for the Closed-Loop Control of Soft Robotic Systems", Soft Robotics, vol. 4, pp. 261-273, 2017.

[11] P. Wu, W. Jiangbei, and F. Yanqiong, "The Structure, Design, and Closed-Loop Motion Control of a Differential Drive Soft Robot", Soft Robotics, vol. 5, pp. 71-80, 2018.

[12] S. Kumbay Yildiz, R. Mutlu, and G. Alici, "Fabrication and characterisation of highly stretchable elastomeric strain sensors for prosthetic hand applications," Sensors and Actuators A: Physical, vol. 247, pp. 514-521, 8/15/ 2016

[13] S. K. Yildiz, R. Mutlu, and G. Alici, "Performance quantification of strain sensors for flexible manipulators," in 2016 IEEE International Conf. on Advanced Intelligent Mechatronics (AIM), 2016, pp. 584-589.

[14] L. K. Simone et al., "A low cost instrumented glove for extended monitoring and functional hand assessment," Journal of Neuroscience Methods, vol. 160, pp. 335-348, 3/15/ 2007.

[15] G. Saggio, "A novel array of flex sensors for a goniometric glove," Sensors and Actuators A: Physical, vol. 205, pp. 119-125, 1/1/ 2014.

[16] L. Sbernini, A. Pallotti, and G. Saggio, "Evaluation of a Stretch Sensor for its inedited application in tracking hand finger movements," in 2016 IEEE International Symposium on Medical Measurements and Applications (MeMeA), 2016, pp. 1-6.

[17] R. Deimel and O. Brock, "A novel type of compliant and underactuated robotic hand for dexterous grasping," The International Journal of Robotics Research, August 21, 20152015.

[18] Y. She, C. Li, J. Cleary, and H.-J. Su, "Design and Fabrication of a Soft Robotic Hand with Embedded Actuators and Sensors," Journal of Mechanisms and Robotics, vol. 7, pp. 021007-021007, 2015.

[19] M. Calisti et al., "An octopus-bioinspired solution to movement and manipulation for soft robots," Bioinspiration \& Biomimetics, vol. 6, p. 036002, 2011.

[20] A. D. Marchese and D. Rus, "Design, kinematics, and control of a soft spatial fluidic elastomer manipulator," The International Journal of Robotics Research, October 6, 20152015.

[21] R. Mutlu, G. Alici, M. in het Panhuis, and G. M. Spinks, "3D Printed Flexure Hinges for Soft Monolithic Prosthetic Fingers," Soft Robotics, vol. 3, pp. 120-133, 2016/09/01 2016.

[22] Ş. K. Yıldız and H. Demircioğlu, "Relay sliding mode control based on the input-output model," Turkish Journal of Electrical Engineering \& Computer Sciences, vol. 24, pp. 2609-2626, 2016.

[23] Ş. K. Yıldız, "Giriș çıkıș modeline dayalı röle ile kayan kipli denetim" (in Turkish), PhD Thesis, Hacettepe University, Ankara, Turkey, 2013.

[24] R. Mutlu, G. Alici, M. in het Panhuis, and G. Spinks, "Effect of flexure hinge type on a 3D printed fully compliant prosthetic finger," in IEEE International Conference on Advanced Intelligent Mechatronics (AIM), 2015, pp. 790-795.

[25] R. Mutlu et al., "Mechanical stiffness augmentation of a 3D printed soft prosthetic finger," in 2016 IEEE International Conference on Advanced Intelligent Mechatronics (AIM), 2016, pp. 7-12.

[26] H. W. Kay and M. Rakic, "Specifications for electromechanical hands," in 4th International Symposium on the External Control of Human Extremities, Belgrade, Yugoslavia, 1972, pp. 137-155.

[27] V. Sencadas, R. Mutlu, and G. Alici, "Large area and ultra-thin compliant strain sensors for prosthetic devices", Sensors and Actuators: A, Physical, Vol 266, pp. 56 - 64, October 2017. 